IFT-P.039/2001

\title{
Covariant Quantization of the Superparticle using Pure Spinors
}

\author{
Nathan Berkovitd] \\ Instituto de Física Teórica, Universidade Estadual Paulista \\ Rua Pamplona 145, 01405-900, São Paulo, SP, Brasil
}

\begin{abstract}
The ten-dimensional superparticle is covariantly quantized by constructing a BRST operator from the fermionic Green-Schwarz constraints and a bosonic pure spinor variable. This same method was recently used for covariantly quantizing the superstring, and it is hoped that the simpler case of the superparticle will be useful for those who want to study this quantization method. It is interesting that quantization of the superparticle action closely resembles quantization of the worldline action for Chern-Simons theory.
\end{abstract}

May 2001

\footnotetext{
1 e-mail: nberkovi@ift.unesp.br
} 


\section{Introduction}

Recently, the ten-dimensional superstring was covariantly quantized by constructing a BRST operator from the fermionic Green-Schwarz constraints and a bosonic pure spinor variable [1]. Although this method was successfully used for computing tree-level scattering amplitudes, the construction of the BRST operator is non-conventional so it is a bit mysterious why this method works. This same BRST construction can be used for covariantly quantizing the ten-dimensional superparticle and it is hoped that by studying this simpler model, some of the mysteries will be easier to understand.

Since the spectrum of the ten-dimensional superparticle contains a spin-one field, the constraints of the worldline action should imply spacetime gauge invariances as well as spacetime equations of motion. This differs from the worldline actions for the particle or spinning particle where the constraints imply the Klein-Gordon or Dirac equations of motion, but do not imply spacetime gauge invariances. One worldline action which does describe a theory with spacetime gauge invariance is the worldline version of Witten's action for Chern-Simons theory [2]. It will turn out that the constraints and quantization of this Chern-Simons action closely resemble the constraints and quantization of the pure spinor version of the superparticle action 2 .

Section 2 of this paper will review the problems with quantizing the standard superparticle action. In section 3, the worldline action for Chern-Simons will be discussed. Section 4 will review the superspace description of ten-dimensional super-Yang-Mills. And in section 5, the pure spinor version of the superparticle action will be quantized in a manner similar to the Chern-Simons action. The appendix will contain a computation of the zero-momentum BRST cohomology of the superparticle.

\section{Review of Standard Superparticle Description}

The standard action for the ten-dimensional superparticle is [3]

$$
S=\int d \tau\left(\Pi^{m} P_{m}+e P^{m} P_{m}\right)
$$

where

$$
\Pi^{m}=\dot{x}^{m}-\frac{i}{2} \dot{\theta}^{\alpha} \gamma_{\alpha \beta}^{m} \theta^{\beta}
$$

2 The similarity of the two worldline actions was first pointed out to me by Warren Siegel. 
$P_{m}$ is the canonical momentum for $x^{m}$, and $e$ is the Lagrange multiplier which enforces the mass-shell condition. The gamma matrices $\gamma_{\alpha \beta}^{m}$ and $\gamma_{m}^{\alpha \beta}$ are $16 \times 16$ symmetric matrices which satisfy $\gamma_{\alpha \beta}^{(m} \gamma^{n) \beta \gamma}=2 \eta^{m n} \delta_{\alpha}^{\gamma}$. In the Weyl representation, $\gamma_{\alpha \beta}^{m}$ and $\gamma_{m}^{\alpha \beta}$ are the off-diagonal blocks of the $32 \times 32 \Gamma^{m}$ matrices.

The action of (2.1) is spacetime-supersymmetric under

$$
\delta \theta^{\alpha}=\epsilon^{\alpha}, \quad x^{m}=\frac{i}{2} \theta \gamma^{m} \epsilon, \quad \delta P_{m}=\delta e=0,
$$

and is also invariant under the local $\kappa$ transformations [ [⿴囗十

$$
\delta \theta^{\alpha}=P^{m}\left(\gamma_{m} \kappa\right)^{\alpha}, \quad \delta x^{m}=-\frac{i}{2} \theta \gamma^{m} \delta \theta, \quad \delta P_{m}=0, \quad \delta e=i \dot{\theta}^{\beta} \kappa_{\beta} .
$$

The canonical momentum to $\theta^{\alpha}$, which will be called $p_{\alpha}$, satisfies

$$
p_{\alpha}=\delta L / \delta \dot{\theta}^{\alpha}=-\frac{i}{2} P^{m}\left(\gamma_{m} \theta\right)_{\alpha},
$$

so canonical quantization requires that physical states are annihilated by the fermionic Dirac constraints defined by

$$
d_{\alpha}=p_{\alpha}+\frac{i}{2} P_{m}\left(\gamma^{m} \theta\right)_{\alpha} .
$$

Since $\left\{p_{\alpha}, \theta^{\beta}\right\}=-i \delta_{\alpha}^{\beta}$, these constraints satisfy the Poisson brackets

$$
\left\{d_{\alpha}, d_{\beta}\right\}=P_{m} \gamma_{\alpha \beta}^{m},
$$

and since $P^{m} P_{m}=0$ is also a constraint, eight of the sixteen Dirac constraints are first-class and eight are second-class. One can easily check that the eight first-class Dirac constraints generate the $\kappa$ transformations of (2.3), however, there is no simple way to covariantly separate out the second-class constraints.

Nevertheless, one can easily quantize the superparticle in a non-Lorentz covariant manner and obtain the physical spectrum. Assuming non-zero $P^{+}$, the local fermionic $\kappa$-transformations can be used to gauge-fix $\left(\gamma^{+} \theta\right)_{\alpha}=0$ where $\gamma^{ \pm}=\frac{1}{\sqrt{2}}\left(\gamma^{0} \pm \gamma^{9}\right)$. In this gauge, the action of (2.1) simplifies to the quadratic action [5]

$$
S=\int d \tau\left(\dot{x}^{m} P_{m}+\frac{i}{2} P^{+}\left(\dot{\theta} \gamma^{-} \theta\right)+e P^{m} P_{m}\right)=\int d \tau\left(\dot{x}^{m} P_{m}+\frac{i}{4} \dot{\sigma}_{a} \sigma_{a}+e P^{m} P_{m}\right),
$$

where $\sigma_{a}=\sqrt{2 P^{+}}\left(\gamma^{-} \theta\right)_{a}$ and $a=1$ to 8 is an $S O(8)$ chiral spinor index. 
Canonical quantization of (2.6) implies that $\left\{\sigma_{a}, \sigma_{b}\right\}=2 \delta_{a b}$. So $\sigma_{a}$ acts like a 'spinor' version of the $S O(8)$ Pauli matrices $\sigma_{a b}^{j}$ which satisfy

$$
\sigma_{a \dot{c}}^{j} \sigma_{b \dot{d}}^{j}+\sigma_{b \dot{c}}^{j} \sigma_{a \dot{d}}^{j}=2 \delta_{a b} \delta_{\dot{c} \dot{d}}
$$

where $j$ and $\dot{b}$ are $S O(8)$ vector and antichiral spinor indices. One can therefore define the quantum-mechanical wavefunction $\Psi(x)$ to carry either an $S O(8)$ vector index, $\Psi_{j}(x)$, or an $S O(8)$ antichiral spinor index, $\Psi_{\dot{a}}(x)$, and the anticommutation relations of $\sigma_{a}$ are reproduced by defining

$$
\sigma^{a} \Psi_{j}(x)=\sigma_{j}^{a \dot{b}} \Psi_{\dot{b}}(x), \quad \sigma_{a} \Psi_{\dot{b}}(x)=\sigma_{a \dot{b}}^{j} \Psi_{j}(x) .
$$

Furthermore, the constraint $P_{m} P^{m}$ implies the linearized equations of motion $\partial_{m} \partial^{m} \Psi_{j}=$ $\partial_{m} \partial^{m} \Psi_{\dot{b}}=0$.

So the physical states of the superparticle are described by a massless $S O(8)$ vector $\Psi_{j}(x)$ and a massless $S O(8)$ antichiral spinor $\Psi_{\dot{a}}(x)$ which are the physical states of $d=10$ super-Yang-Mills theory. However, this description of super-Yang-Mills theory only manifestly preserves an $S O(8)$ subgroup of the super-Poincaré group, and one would like a more covariant method for quantizing the theory. Covariant quantization can be extremely useful if one wants to compute more than just the physical spectrum in a flat background. For example, non-covariant methods are extremely clumsy for computing scattering amplitudes or for generalizing to curved backgrounds.

Since the super-Yang-Mills spectrum contains a massless vector, one expects the covariant superparticle constraints to generate the spacetime gauge invariances of this vector. Note that these constraints are not present in the gauge-fixed action of (2.6) since $\Psi_{j}$ describes only the transverse degrees of freedom of the $S O(9,1)$ vector. Before describing the covariant constraints which generate the gauge invariances of this vector, it will be useful to first review the worldline action for Chern-Simons theory which also has constraints related to spacetime gauge invariances.

\section{Worldline Description of Chern-Simons Theory}

Since the gauge invariance of a massless vector field is $\delta A_{\mu}=\partial_{\mu} \Lambda$, one might guess that the worldline action for such a field should contain the constraints $P_{\mu}$. Although these constraints are too strong for describing Yang-Mills theory, they are just right for describing $d=3$ Chern-Simons theory where the field-strength of $A_{\mu}$ vanishes on-shell. 


\subsection{Worldline action}

As was shown in [2], Chern-Simons theory can be described using the worldline action

$$
S=\int d \tau\left(\dot{x}^{\mu} P_{\mu}+l^{\mu} P_{\mu}\right)
$$

where $\mu=0$ to 2 and $l^{\mu}$ are Lagrange multipliers for the constraints. Since the constraints are first-class, the action can be quantized using the BRST method. After gauging $l^{\mu}=$ $-\frac{1}{2} P^{\mu}$, the gauge-fixed action is

$$
S=\int d \tau\left(\dot{x}^{\mu} P_{\mu}-\frac{1}{2} P^{\mu} P_{\mu}+\dot{c}^{\mu} b_{\mu}\right)
$$

with the BRST operator

$$
Q=c^{\mu} P_{\mu}
$$

where $\left(c^{\mu}, b_{\mu}\right)$ are fermionic Fadeev-Popov ghosts and anti-ghosts.

To show that the cohomology of the BRST operator describes Chern-Simons theory, note that the most general wavefunction constructed from a ground state annihilated by $b^{\mu}$ is

$$
\Psi(c, x)=C(x)+c^{\mu} A_{\mu}(x)+\frac{i}{2} \epsilon_{\mu \nu \rho} c^{\mu} c^{\nu} A^{* \rho}(x)+\frac{i}{6} \epsilon_{\mu \nu \rho} c^{\mu} c^{\nu} c^{\rho} C^{*}(x)
$$

where the expansion in $c^{\mu}$ terminates since $c^{\mu}$ is fermionic. One can check that

$$
Q \Psi=-i c^{\mu} \partial_{\mu} C-\frac{i}{2} c^{\mu} c^{\nu} \partial_{[\mu} A_{\nu]}+\frac{1}{6} \epsilon_{\mu \nu \rho} c^{\mu} c^{\nu} c^{\rho} \partial_{\sigma} A^{* \sigma}(x) .
$$

So $Q \Psi=0$ implies that $A_{\mu}(x)$ satisfies the equations of motion $\partial_{[\mu} A_{\nu]}=0$ which is the linearized equation of motion of the Chern-Simons field. Furthermore, if one defines the gauge parameter $\Omega(c, x)=i \Lambda(x)-c^{\mu} \omega_{\mu}(x)+\ldots$, the gauge transformation $\delta \Psi=Q \Omega$ implies $\delta A_{\mu}=\partial_{\mu} \Lambda$ which is the linearized gauge transformation of the Chern-Simons field. Similarly, $Q \Psi=0$ and $\delta \Psi=Q \Omega$ implies that $A^{* \rho}$ satisfies the equation of motion $\partial_{\sigma} A^{* \sigma}=0$ with the gauge invariance $\delta A^{* \sigma}=\epsilon^{\sigma \mu \nu} \partial_{\mu} w_{\nu}$, which are the linearized equations of motion and gauge invariance of the Chern-Simons antifield. The remaining fields, $C(x)$ and $C^{*}(x)$, describe the spacetime ghost and antighost of Chern-Simons theory.

These equations of motion and gauge invariances can be obtained from the BatalinVilkovisky version [6] of the abelian Chern-Simons spacetime action

$$
\mathcal{S}=\int d^{3} x\left(\frac{1}{2} \epsilon^{\mu \nu \rho} A_{\mu} \partial_{\nu} A_{\rho}+i A^{* \mu} \partial_{\mu} C\right),
$$

where, in addition to the usual Chern-Simons action for $A_{\mu}$, there is a term coupling the antifield $A^{* \mu}$ to the gauge variation of $A_{\mu}$. The action of (3.6) can be written compactly in terms of the wavefunction $\Psi$ of (3.4) as

$$
\mathcal{S}=\frac{1}{2} \int d^{3} x\langle\Psi Q \Psi\rangle
$$

where \langle\rangle is normalized such that $\left\langle c^{\mu} c^{\nu} c^{\rho}\right\rangle=i \epsilon^{\mu \nu \rho}$. 


\subsection{Non-abelian Chern-Simons theory}

Up to now, only abelian Chern-Simons theory has been discussed, but it is easy to generalize to the non-abelian case. For example, the Batalin-Vilkovisky version of the non-abelian Chern-Simons action is

$$
\begin{aligned}
\mathcal{S}= & \operatorname{Tr} \int d^{3} x\left(\epsilon^{\mu \nu \rho}\left(\frac{1}{2} A_{\mu} \partial_{\nu} A_{\rho}+\frac{i g}{3} A_{\mu} A_{\nu} A_{\rho}\right)\right. \\
& \left.+i A^{* \mu}\left(\partial_{\mu} C+i g\left[A_{\mu}, C\right]\right)-g C C C^{*}\right)
\end{aligned}
$$

which can be written compactly as

$$
\mathcal{S}=\operatorname{Tr} \int d^{3} x\left\langle\frac{1}{2} \Psi Q \Psi+\frac{g}{3} \Psi \Psi \Psi\right\rangle
$$

where $g$ is the Chern-Simons coupling constant and the fields in $\Psi$ of (3.4) now carry Lie algebra indices. 3 Note that the non-linear equations of motion and gauge invariances associated with this action are

$$
Q \Psi+g \Psi \Psi=0, \quad \delta \Psi=Q \Omega+g[\Omega, \Psi]
$$

To construct a wordline action for non-abelian Chern-Simons theory with $S O(N)$ gauge group, one introduces $N$ real fermionic variables $\eta_{I}$ for $I=1$ to $N$ and modifies the worldline action of (3.2) tot

$$
S=\int d \tau\left(\dot{x}^{\mu} P_{\mu}-\frac{1}{2} P^{\mu} P_{\mu}-\frac{i}{2} \eta_{I} \nabla \eta_{I}+\dot{c}^{\mu} b_{\mu}\right)
$$

where $\nabla \eta_{I}=\dot{\eta}_{I}+g \eta_{J} \dot{x}^{\mu} \tilde{A}_{\mu}^{I J}(x)$ and $\tilde{A}_{\mu}^{I J}(x)=-\tilde{A}_{\mu}^{J I}(x)$ is a non-abelian Chern-Simons background field [7]. Note that the constraints $P_{\mu}$ are conserved when the background is on-shell since

$$
\dot{P}_{\mu}=\frac{i g}{2}\left(\frac{\partial}{\partial \tau}\left(\eta_{I} \eta_{J} \tilde{A}_{\mu}^{I J}\right)-\eta_{I} \eta_{J} \dot{x}^{\nu} \partial_{\mu} \tilde{A}_{\nu}^{I J}\right)
$$

3 It is interesting to note that the Chern-Simons action can also be written in manifestly gaugeinvariant notation as $\mathcal{S}=\frac{1}{2} \operatorname{Tr} \int_{\mathcal{M}} d^{4} x\left\langle(Q \Psi+g \Psi \Psi)^{2}\right\rangle_{4}$ where $\mathcal{M}$ is a four-dimensional volume with a three-dimensional boundary at $x_{3}=0, Q=c^{M} P_{M}$ for $M=0$ to $3,\left\langle c^{M} c^{N} c^{P} c^{Q}\right\rangle_{4}=\epsilon^{M N P Q}$, and $\Psi$ now depends on $x^{3}$ and $c^{3}$. Using cyclicity of the trace, one finds $\mathcal{S}=\operatorname{Tr} \int_{\mathcal{M}} d^{4} x\left\langle Q\left(\frac{1}{2} \Psi Q \Psi+\right.\right.$ $\left.\left.\frac{g}{3} \Psi \Psi \Psi\right)\right\rangle_{4}=\operatorname{Tr} \int d^{4} x\left\langle\delta\left(c^{3}\right) \delta\left(x^{3}\right)\left(\frac{1}{2} \Psi Q \Psi+\frac{g}{3} \Psi \Psi \Psi\right)\right\rangle_{4}$ since $\int_{\mathcal{M}} d^{4} x\langle Q \Lambda\rangle_{4}$ only gets contributions from the three-dimensional boundary at $x_{3}=0$. So $\mathcal{S}$ coincides with the Chern-Simons action defined in $(3.9)$.

4 For $U(N)$ gauge group, one introduces $N$ complex fermionic variables $\left(\eta_{I}, \bar{\eta}^{I}\right)$ with the action $-i \int d \tau \bar{\eta}^{I} \nabla \eta_{I}$ 


$$
=-\frac{i g}{2} \eta_{I} \eta_{J} \dot{x}^{\nu}\left(\partial_{[\mu} \tilde{A}_{\nu]}^{I J}+g \tilde{A}_{[\mu}^{I K} \tilde{A}_{\nu]}^{K J}\right)=0 .
$$

After gauge-fixing, the BRST charge is still $Q=c^{\mu} P_{\mu}$, but because of the background gauge field $\tilde{A}_{\mu}^{I J}$ in $(3.11)$, the canonical momentum for $x^{\mu}$ is now $\frac{\partial L}{\partial \dot{x}^{\mu}}=P_{\mu}-\frac{i g}{2} \eta_{I} \eta_{J} \tilde{A}_{\mu}^{I J}$. Since $\left\{\eta_{I}, \eta_{J}\right\}=\delta_{I J}$,

$$
Q\left(\eta_{I} \eta_{J} \Psi^{I J}\right)=\eta_{I} \eta_{J}\left(-i c^{\mu}\left(\nabla_{\mu} C\right)^{I J}-\frac{i}{2} c^{\mu} c^{\nu}\left(\nabla_{[\mu} A_{\nu]}\right)^{I J}+\frac{1}{6} \epsilon_{\mu \nu \rho} c^{\mu} c^{\nu} c^{\rho}\left(\nabla_{\sigma} A^{* \sigma}\right)^{I J}\right)
$$

where $\left(\nabla_{\mu} s\right)^{I J}=\partial_{\mu} s^{I J}+g\left(s^{I K} \tilde{A}_{\mu}^{K J}-s^{J K} \tilde{A}_{\mu}^{K I}\right)$ and $\Psi^{I J}$ is defined as in (3.4). So in a background gauge field $\tilde{A}_{\mu}^{I J}$, the linearized equations of motion of the non-abelian ChernSimons field and antifield are correctly described by $Q\left(\eta_{I} \eta_{J} \Psi^{I J}\right)=0$.

Using intuition learned from this worldline description of Chern-Simons theory, it will be shown how to quantize the superparticle in a similar manner. However, before performing this quantization, it will be useful to first review the superspace description of ten-dimensional super-Yang-Mills theory.

\section{Covariant Description of Super-Yang-Mills Theory}

Although on-shell super-Yang-Mills theory can be described by the $S O(8)$ wavefunctions $\Psi_{j}(x)$ and $\Psi_{\dot{a}}(x)$ of (2.7) satisfying the linearized equations of motion $\partial_{m} \partial^{m} \Psi_{j}=$ $\partial_{m} \partial^{m} \Psi_{\dot{a}}=0$, there are more covariant descriptions of the theory. Of course, there is a Poincaré-covariant description using an $S O(9,1)$ vector field $a_{m}(x)$ and an $S O(9,1)$ spinor field $\chi^{\alpha}(x)$ transforming in the adjoint representation of the gauge group which satisfy the equations of motion

$$
\partial^{m} f_{m n}+i g\left[a^{m}, f_{m n}\right]=0, \quad \gamma_{\alpha \beta}^{m}\left(\partial_{m} \chi^{\beta}+i g\left[a_{m}, \chi^{\beta}\right]\right)=0
$$

and gauge invariance

$$
\delta a_{m}=\partial_{m} s+i g\left[a_{m}, s\right], \quad \delta \chi^{\alpha}=i g\left[\chi^{\alpha}, s\right], \quad \delta f_{m n}=i g\left[f_{m n}, s\right]
$$

where $f_{m n}=\partial_{[m} a_{n]}+i g\left[a_{m}, a_{n}\right]$ is the Yang-Mills field strength and $g$ is the super-YangMills coupling constant. However, there is also a super-Poincaré covariant description using an $S O(9,1)$ spinor wavefunction $A_{\alpha}(x, \theta)$ defined in $d=10$ superspace. As will be explained below, on-shell super-Yang-Mills theory can be described by a spinor superfield 
$A_{\alpha}(x, \theta)$ transforming in the adjoint representation which satisfies the superspace equation of motion [8]

$$
\gamma_{m n p q r}^{\alpha \beta}\left(D_{\alpha} A_{\beta}+i g A_{\alpha} A_{\beta}\right)=0
$$

for any five-form direction mnpqr, with the gauge invariance

$$
\delta A_{\alpha}=D_{\alpha} \Lambda+i g\left[A_{\alpha}, \Lambda\right]
$$

where $\Lambda(x, \theta)$ is any scalar superfield and

$$
D_{\alpha}=\frac{\partial}{\partial \theta^{\alpha}}+\frac{i}{2}\left(\gamma^{m} \theta\right)_{\alpha} \partial_{m}
$$

is the supersymmetric derivative.

One can also define field strengths constructed from $A_{\alpha}$ by

$$
\begin{gathered}
B_{m}=-\frac{i}{8} \gamma_{m}^{\alpha \beta}\left(D_{\alpha} A_{\beta}+i g A_{\alpha} A_{\beta}\right), \quad W^{\alpha}=\frac{1}{10} \gamma_{m}^{\alpha \beta}\left(D_{\alpha} B^{m}-\partial^{m} A_{\alpha}+i g\left[A_{\alpha}, B^{m}\right]\right), \\
F_{m n}=\partial_{[m} B_{n]}+i g\left[B_{m}, B_{n}\right]=\frac{1}{8}\left(\gamma_{m n}\right)_{\alpha}^{\beta}\left(D_{\beta} W^{\alpha}+i g\left\{A_{\beta}, W^{\alpha}\right\}\right) .
\end{gathered}
$$

Under the gauge transformation of (4.4),

$$
\delta B_{m}=\partial_{m} \Lambda+i g\left[B_{m}, \Lambda\right], \quad \delta W^{\alpha}=i g\left[W^{\alpha}, \Lambda\right], \quad \delta F^{m n}=i g\left[F^{m n}, \Lambda\right] .
$$

To show that $A_{\alpha}(x, \theta)$ describes on-shell super-Yang-Mills theory, it will be useful to first note that in ten dimensions any symmetric bispinor $f_{\alpha \beta}$ can be decomposed in terms of a vector and a five-form as $f_{\alpha \beta}=\gamma_{\alpha \beta}^{m} f_{m}+\gamma_{\alpha \beta}^{m n p q r} f_{m n p q r}$ and any antisymmetric bispinor $f_{\alpha \beta}$ can be decomposed in terms of a three-form as $f_{\alpha \beta}=\gamma_{\alpha \beta}^{m n p} f_{m n p}$. Since $\left\{D_{\alpha}, D_{\beta}\right\}=$ $i \gamma_{\alpha \beta}^{m} \partial_{m}$, one can check that $\delta A_{\alpha}=D_{\alpha} \Lambda+i g\left[A_{\alpha}, \Lambda\right]$ is indeed a gauge invariance of (4.3).

Using $\Lambda(x, \theta)=h_{\alpha}(x) \theta^{\alpha}+j_{\alpha \beta}(x) \theta^{\alpha} \theta^{\beta}$, one can gauge away $\left.\left(A_{\alpha}(x)\right)\right|_{\theta=0}$ and the threeform part of $\left.\left(D_{\alpha} A_{\beta}(x)\right)\right|_{\theta=0}$. Furthermore, equation (4.3) implies that the five-form part of $\left.\left(D_{\alpha} A_{\beta}(x)\right)\right|_{\theta=0}$ vanishes. So the lowest non-vanishing component of $A_{\alpha}(x, \theta)$ in this gauge is the vector component $\left.\left(D \gamma_{m} A(x)\right)\right|_{\theta=0}$ which will be defined as $8 i a_{m}(x)$. Continuing this type of argument to higher order in $\theta^{\alpha}$, one finds that there exists a gauge choice such that

$$
A_{\alpha}(x, \theta)=\frac{i}{2}\left(\gamma^{m} \theta\right)_{\alpha} a_{m}(x)+\frac{i}{12}\left(\theta \gamma^{m n p} \theta\right)\left(\gamma_{m n p}\right)_{\alpha \beta} \chi^{\beta}(x)+\ldots
$$

where $a_{m}(x)$ and $\chi^{\beta}(x)$ are $S O(9,1)$ vector and spinor fields satisfying (4.1) and where the component fields in $\ldots$ are functions of spacetime derivatives of $a_{m}(x)$ and $\chi^{\beta}(x)$. Furthermore, this gauge choice leaves the residual gauge transformations of (4.2) where $s(x)=\left.(\Lambda(x))\right|_{\theta=0}$. Also, one can check that the $\theta=0$ components of the superfields $B_{m}$, $W^{\alpha}$ and $F_{m n}$ of (4.5) are $a_{m}, \chi^{\alpha}$ and $f_{m n}$ respectively. So the equations of motion and gauge invariances of (4.3) and (4.4) correctly describe on-shell super-Yang-Mills theory.

One would now like to obtain this super-Poincaré covariant description of super-YangMills theory by quantizing the superparticle. As will now be shown, this can be done by constructing a BRST-like operator out of the fermionic constraints $d_{\alpha}$ of (2.4). 


\section{Covariant Quantization of the Superparticle}

In the case of Chern-Simons theory, the gauge transformation $\delta A_{\mu}=\partial_{\mu} \Lambda$ was generated by the constraints $P_{\mu}$. So for the superparticle, the gauge transformation $\delta A_{\alpha}=D_{\alpha} \Lambda$ suggests using the constraints $d_{\alpha}$. However, the constraints $d_{\alpha}$ are not all first-class, so

$$
Q=\lambda^{\alpha} d_{\alpha}
$$

would not be a nilpotent operator for generic $\lambda^{\alpha}$. However, since (2.5) implies that $Q^{2}=$ $\left(\lambda^{\alpha} d_{\alpha}\right)^{2}=\frac{1}{2} \lambda^{\alpha} \lambda^{\beta} \gamma_{\alpha \beta}^{m} P_{m}, Q$ would be nilpotent if $\lambda^{\alpha}$ satisfied the condition

$$
\lambda^{\alpha} \gamma_{\alpha \beta}^{m} \lambda^{\beta}=0
$$

for $m=0$ to 9 . The condition of (5.2) is the definition of a pure spinor [9] and, as will now be shown, implies that only eleven components of $\lambda^{\alpha}$ are independent parameters.

\subsection{Pure spinors}

To solve the constraint of (5.2), it is convenient to first Wick-rotate to Euclidean space and write the $S O(10)$ spinor $\lambda^{\alpha}$ using $S U(5)$ notation as $\lambda^{ \pm \pm \pm \pm \pm}$where \pm denotes if the component is annihilated by $\left(\gamma_{2 a-2}+i \gamma_{2 a-1}\right)$ or $\left(\gamma_{2 a-2}-i \gamma_{2 a-1}\right)$ for $a=1$ to 5 . For example, the component $\lambda^{+-+-+}$is annihilated by $\gamma_{0}+i \gamma_{1}, \gamma_{2}-i \gamma_{3}, \gamma_{4}+i \gamma_{5}, \gamma_{6}-i \gamma_{7}$, and $\gamma_{8}+i \gamma_{9}$. For a sixteen-component Weyl spinor, $\lambda^{ \pm \pm \pm \pm \pm}$contains either five + 's, three + 's, or one + , which transform respectively under $S U(5)$ as $1, \overline{10}$, and 5 representations. These $S U(5)$ representations will be called $\lambda^{+}, \lambda_{a b}$ and $\lambda^{a}$ where $a=1$ to 5 and $\lambda_{a b}=-\lambda_{b a}$. One can check that any pure spinor $\lambda^{\alpha}$ satisfying (5.2) can be parameterized as

$$
\lambda^{+}=\gamma, \quad \lambda_{a b}=\gamma u_{a b}, \quad \lambda^{a}=-\frac{\gamma}{8} \epsilon^{a b c d e} u_{b c} u_{d e}
$$

where $u_{a b}=-u_{b a}$ and $\gamma$ are eleven independent parameters. To show that (5.3) satisfies (5.2), note that

$$
\lambda \gamma^{a} \lambda=\lambda^{+} \lambda^{a}+\frac{1}{8} \epsilon^{a b c d e} \lambda_{b c} \lambda_{d e}, \quad \lambda \gamma_{a} \lambda=\lambda^{b} \lambda_{a b}
$$

where $\gamma^{a}=\frac{1}{\sqrt{2}}\left(\gamma_{2 a-2}-i \gamma_{2 a-1}\right)$ and $\gamma_{a}=\frac{1}{\sqrt{2}}\left(\gamma_{2 a-2}+i \gamma_{2 a-1}\right)$. Also note that the parameterization of (5.3) is singular when $\lambda^{+}=0$ since $\gamma \rightarrow 0$ and $u_{a b} \rightarrow \infty$ when $\lambda^{+} \rightarrow 0$.

To obtain the BRST operator of (5.1) from gauge-fixing, start with the worldline action

$$
S=\int d \tau\left(\dot{x}^{m} P_{m}-\frac{1}{2} P^{m} P_{m}+\dot{\theta}^{\alpha} p_{\alpha}+\frac{1}{2} \dot{u}_{a b} v^{a b}+l\left(\lambda^{\alpha} / \gamma\right) d_{\alpha}\right)
$$


where $p_{\alpha}$ is the conjugate momentum for $\theta^{\alpha}, v^{a b}$ is the conjugate momentum for $u_{a b}, \lambda^{\alpha} / \gamma$ is defined in terms of $u_{a b}$ using the definition of (5.3), and $l$ is the Lagrange multiplier for the constraint $\left(\lambda^{\alpha} / \gamma\right) d_{\alpha}$. 5 After gauge fixing $l=0$, one obtains the action

$$
S=\int d \tau\left(\dot{x}^{m} P_{m}-\frac{1}{2} P^{m} P_{m}+\dot{\theta}^{\alpha} p_{\alpha}+\frac{1}{2} \dot{u}_{a b} v^{a b}+\dot{\gamma} \beta\right)
$$

with the BRST operator $Q=\lambda^{\alpha} d_{\alpha}$ where $(\gamma, \beta)$ are the bosonic Fadeev-Popov ghost and antighost for the constraint $\left(\lambda^{\alpha} / \gamma\right) d_{\alpha}$.

Note that the action and BRST operator of (5.6) and (5.1) are spacetime supersymmetric since $\dot{x}^{m} P_{m}+\dot{\theta}^{\alpha} p_{\alpha}=\Pi^{m} P_{m}+\dot{\theta}^{\alpha} d_{\alpha}$ where $\Pi^{m}$ and $d_{\alpha}$ are defined in (2.2) and (2.4). Furthermore, although $(\gamma, \beta)$ and $\left(u_{a b}, v^{a b}\right)$ do not transform linearly under Lorentz transformations, one can define Lorentz generators such that the pure spinor $\lambda^{\alpha}$ of (5.3) does transform linearly as $\delta \lambda^{\alpha}=\frac{1}{2}\left(\gamma^{m n}\right)^{\alpha}{ }_{\beta} \lambda^{\beta}$ under the transformation generated by $N^{m n}$. These Lorentz generators are given by

$$
\begin{gathered}
N^{a b}=v^{a b}, \quad N_{a b}=u_{a c} u_{b d} v^{c d}-u_{a b} \gamma \beta, \\
N_{b}^{a}=u_{b c} v^{a c}-\frac{1}{5} \delta_{b}^{a} u_{c d} v^{c d}, \quad N=\frac{5}{2} \gamma \beta-u_{a b} v^{a b},
\end{gathered}
$$

where the $45 S O(10)$ Lorentz generators $N^{m n}$ have been decomposed in terms of their irreducible $S U(5)$ representations $\left(N^{a b}, N_{a b}, N_{b}^{a}, N\right)$ which transform as $(10, \overline{10}, 24,1)$ representations. So the action and BRST operator of (5.6) and (5.1) are super-Poincaré invariant and it will now be shown that they correctly describe super-Yang-Mills theory.

\subsection{Quantization}

The most general super-Poincaré covariant wavefunction that can be constructed from $\left(x^{m}, \theta^{\alpha}, \lambda^{\alpha}\right)$ is 6

$$
\Psi(x, \theta, \lambda)=C(x, \theta)+\lambda^{\alpha} A_{\alpha}(x, \theta)+\left(\lambda \gamma^{m n p q r} \lambda\right) A_{m n p q r}^{*}(x, \theta)+\lambda^{\alpha} \lambda^{\beta} \lambda^{\gamma} C_{\alpha \beta \gamma}^{*}(x, \theta)+\ldots
$$

where $\ldots$ includes superfields with more than three powers of $\lambda^{\alpha}$. Since $Q \Psi=$ $-i \lambda^{\alpha} D_{\alpha} C-i \lambda^{\alpha} \lambda^{\beta} D_{\alpha} A_{\beta}+\ldots, Q \Psi=0$ implies that $A_{\alpha}(x, \theta)$ satisfies the equation of

${ }^{5}$ Note that the term $-\frac{1}{2} P^{m} P_{m}$ appears before gauge-fixing, implying that the action of (5.5) is not invariant under worldline reparameterizations. This fact is probably related to the indirect manner in which BRST invariance imposes the mass-shell condition.

6 It will be assumed that $\Psi$ transforms covariantly under Lorentz transformations, which implies that it only depends on $\gamma$ and $u_{a b}$ through the combination $\lambda^{\alpha}$ of (5.3). 
motion $\lambda^{\alpha} \lambda^{\beta} D_{\alpha} A_{\beta}=0$. But since $\lambda^{\alpha} \lambda^{\beta}$ is proportional to $\left(\lambda \gamma^{m n p q r} \lambda\right) \gamma_{m n p q r}^{\alpha \beta}$, this implies that $D \gamma^{m n p q r} A=0$, which is the linearized version of the super-Yang-Mills equation of motion of (4.3). Furthermore, if one defines the gauge parameter $\Omega=i \Lambda+\lambda^{\alpha} \omega_{\alpha}+\ldots$, the gauge transformation $\delta \Psi=Q \Omega$ implies $\delta A_{\alpha}=D_{\alpha} \Lambda$ which is the linearized super-YangMills gauge transformation of (4.4).

As was shown in [10], the only states at non-zero momentum in the cohomology of $Q$ are the on-shell super-Yang-Mills gluon and gluino, $a_{m}(x)$ and $\chi^{\alpha}(x)$, and their antifields, $a^{* m}(x)$ and $\chi_{\alpha}^{*}(x)$ 日. Since gauge invariances of the antifields correspond to equations of motion of the fields and vice versa, one expects $a^{* m}$ and $\chi_{\alpha}^{*}$ to satisfy the linearized equations of motion $\partial_{m} a^{* m}=0$ with the linearized gauge invariances

$$
\delta a^{* m}=\partial_{n}\left(\partial^{n} s^{m}-\partial^{m} s^{n}\right), \quad \delta \chi_{\alpha}^{*}=\gamma_{\alpha \beta}^{m} \partial_{m} \kappa^{\beta}
$$

where $s^{m}$ and $\kappa^{\beta}$ are gauge parameters.

The fields $a_{m}$ and $\chi^{\alpha}$ appear in components of $A_{\alpha}$ as in (4.7), and the antifields $a^{* m}$ and $\chi_{\alpha}^{*}$ appear in components of the ghost-number +2 superfield $A_{m n p q r}^{*}$ of (5.8). Using $Q \Psi=0$ and $\delta \Psi=Q \Omega, A_{m n p q r}^{*}$ satisfies the linearized equation of motion $\lambda^{\alpha}\left(\lambda \gamma^{m n p q r} \lambda\right) D_{\alpha} A_{m n p q r}^{*}=0$ with the linearized gauge invariance $\delta A_{m n p q r}^{*}=\gamma_{m n p q r}^{\alpha \beta} D_{\alpha} \omega_{\beta}$. Expanding $\omega_{\alpha}$ and $A_{m n p q r}^{*}$ in components, one learns that $A_{m n p q r}^{*}$ can be gauged to the form

$$
A_{m n p q r}^{*}=\left(\theta \gamma_{[m n p} \theta\right)\left(\theta \gamma_{q r]}\right)^{\alpha} \chi_{\alpha}^{*}(x)+\left(\theta \gamma_{[m n p} \theta\right)\left(\theta \gamma_{q r] s} \theta\right) a^{* s}(x)+\ldots
$$

where $\chi_{\alpha}^{*}$ and $a^{* s}$ satisfy the equations of motion and residual gauge invariances of (5.9), and ... involves terms higher order in $\theta^{\alpha}$ which depend on derivatives of $\chi_{\alpha}^{*}$ and $a^{* s}$.

As will be shown in the appendix, there are also zero momentum states in the cohomology of $Q$. In addition to the states described by the zero-momentum gluon, gluino, antigluon, and antigluino, there are also zero-momentum ghost and antighost states $c$ and $c^{*}$ in the $\theta=0$ component of the ghost-number zero superfield, $C(x, \theta)=$ $c(x)+\ldots$, and in the $(\theta)^{5}$ component of the ghost-number +3 superfield, $C_{\alpha \beta \gamma}^{*}(x, \theta)=$ $\ldots+c^{*}(x)\left(\gamma^{m} \theta\right)_{\alpha}\left(\gamma^{n} \theta\right)_{\beta}\left(\gamma^{p} \theta\right)_{\gamma}\left(\theta \gamma_{m n p} \theta\right)+\ldots$. So although $\Psi$ of (5.8) contains superfields of arbitrarily high ghost number, only superfields with ghost-number between zero and three contain states in the cohomology of $Q$.

7 The presence of the antifields can be seen from the doubling of the cohomology at ghostnumbers +1 and +2 . 
The linearized equations of motion and gauge invariances $Q \Psi=0$ and $\delta \Psi=Q \Omega$ are easily generalized to the non-linear equations of motion and gauge invariances

$$
Q \Psi+g \Psi \Psi=0, \quad \delta \Psi=Q \Omega+g[\Psi, \Omega]
$$

where $\Psi$ and $\Omega$ transform in the adjoint representation of the gauge group. For the superfield $A_{\alpha}(x, \theta)$, (5.11) implies the super-Yang-Mills equations of motion and gauge transformations of (4.3) and (4.4). Furthermore, the equations of motion and gauge transformation of (5.11) can be obtained from the spacetime action 8

$$
\mathcal{S}=\operatorname{Tr} \int d^{10} x\left\langle\frac{1}{2} \Psi Q \Psi+\frac{g}{3} \Psi \Psi \Psi\right\rangle
$$

using the normalization definition that

$$
\left\langle\left(\lambda \gamma^{m} \theta\right)\left(\lambda \gamma^{n} \theta\right)\left(\lambda \gamma^{p} \theta\right)\left(\theta \gamma_{m n p} \theta\right)\right\rangle=1
$$

Although (5.13) may seem strange, it resembles the normalization of (3.7) in that $\langle\Psi\rangle=$ $c^{*}(x)$ where $c^{*}(x)$ is the spacetime antighost. 6 After writing (5.12) in terms of component fields and integrating out auxiliary fields, it should be possible to show that (5.12) reduces to the standard Batalin-Vilovisky action for super-Yang-Mills,

$$
\begin{aligned}
\mathcal{S}= & \operatorname{Tr} \int d^{10} x\left(\frac{1}{4} f_{m n} f^{m n}+\chi^{\alpha} \gamma_{\alpha \beta}^{m}\left(\partial_{m} \chi^{\beta}+i g\left[a_{m}, \chi^{\beta}\right]\right)\right. \\
& \left.+i a^{* m}\left(\partial_{m} c+i g\left[a_{m}, c\right]\right)-g \chi_{\alpha}^{*}\left\{\chi^{\alpha}, c\right\}-g c c c^{*}\right) .
\end{aligned}
$$

8 This spacetime action was first proposed to me by John Schwarz and Edward Witten. Because the action only involves integration over five $\theta$ 's, it is not manifestly spacetime supersymmetric. Nevertheless, the equations of motion coming from this action have the same physical content as the manifestly spacetime supersymmetric equations of motion $Q \Psi+g \Psi \Psi=0$. This is because all components in $Q \Psi+g \Psi \Psi=0$ with more than five $\theta$ 's are auxiliary equations of motion. So removing these equations of motion only changes auxiliary fields to gauge fields but does not affect the physical content of the theory. Unfortunately, this does not seem to be true after including the massive modes of the superstring. So there does not appear to exist a cubic superstring field theory action which reproduces the equations of motion $Q \Psi+g \Psi \times \Psi=0$ where $\Psi$ is the superstring field and $\times$ is Witten's midpoint interaction.

9 It would be interesting to try to derive (5.11) from an eleven-dimensional action, in analogy to the four-dimensional Chern-Simons action of footnote 3. One natural guess would be to extend $\lambda$ and $\theta$ to eleven-dimensional spinors and define the non-vanishing normalization as $\left\langle\left(\lambda \gamma^{m} \theta\right)\left(\lambda \gamma^{n} \theta\right)\left(\lambda \gamma^{p} \theta\right)\left(\lambda \gamma^{q} \theta\right)\left(\theta \gamma_{m n p q} \theta\right)\right\rangle=1$. 


\subsection{Non-abelian super-Yang-Mills background}

As in Chern-Simons theory, one can modify the worldline action of (5.6) to describe the superparticle in a non-abelian super-Yang-Mills background with $S O(N)$ (or $U(N)$ ) gauge group by including $N$ real (or complex) fermions. For $S O(N)$ gauge group, the worldline action is

$$
S=\int d \tau\left(\dot{x}^{m} P_{m}-\frac{1}{2} P^{m} P_{m}+\dot{\theta}^{\alpha} p_{\alpha}-\frac{i}{2} \eta_{I} \nabla \eta_{I}+\frac{1}{2} \dot{u}_{a b} v^{a b}+\dot{\gamma} \beta\right)
$$

where

$$
\nabla \eta_{I}=\dot{\eta}_{I}+g \eta_{J}\left(\dot{\theta}^{\alpha} \tilde{A}_{\alpha}^{I J}+\Pi^{m} \tilde{B}_{m}^{I J}+d_{\alpha} \tilde{W}^{\alpha I J}+\frac{1}{2} N^{m n} \tilde{F}_{m n}^{I J}\right),
$$

$\tilde{A}_{\alpha}^{I J}$ is the background super-Yang-Mills gauge field, $\tilde{B}_{m}^{I J}, \tilde{W}^{\alpha I J}$ and $\tilde{F}_{m n}^{I J}$ are background superfields constructed from $\tilde{A}_{\alpha}^{I J}$ as in (4.5), $\Pi^{m}$ is defined in (2.2), and $N_{m n}$ is defined in (5.7).

As will now be shown, the coupling of $\tilde{A}_{\alpha}^{I J}$ has been chosen such that $\lambda^{\alpha} d_{\alpha}$ is conserved. To show this, note that $\dot{\lambda}^{\alpha}=-\frac{i g}{8} \eta_{I} \eta_{J}\left(\gamma^{m n} \lambda\right)^{\alpha} F_{m n}^{I J}$ and

$$
\begin{gathered}
\dot{d}_{\alpha}=\frac{i g}{2} \frac{\partial}{\partial \tau}\left(\eta_{I} \eta_{J} \tilde{A}_{\alpha}^{I J}\right)-\frac{i g}{2} \eta_{I} \eta_{J}\left(-\dot{\theta}^{\beta} D_{\alpha} \tilde{A}_{\beta}^{I J}+\Pi^{m} D_{\alpha} \tilde{B}_{m}^{I J}\right. \\
-d_{\beta} D_{\alpha} \tilde{W}^{\beta I J}+\frac{1}{2} N_{m n} D_{\alpha} \tilde{F}_{m n}^{I J}+\gamma_{\alpha \beta}^{m}\left(i \dot{\theta}^{\beta} \tilde{B}_{m}^{I J}+\Pi_{m} \tilde{W}^{\beta I J}\right) \\
=\frac{i g}{2} \eta_{I} \eta_{J}\left(\dot{\theta}^{\beta}\left(D_{(\alpha} \tilde{A}_{\beta)}^{I J}+g \tilde{A}_{(\alpha}^{I K} \tilde{A}_{\beta)}^{K J}-i \gamma_{\alpha \beta}^{m} \tilde{B}_{m}^{I J}\right)+\Pi^{m}\left(-\left(\nabla_{\alpha} \tilde{B}_{m}\right)^{I J}+\partial_{m} \tilde{A}_{\alpha}^{I J}+\gamma_{m} \alpha \beta W^{\beta I J}\right)\right. \\
\left.+d_{\beta}\left(\nabla_{\alpha} \tilde{W}^{\beta}\right)^{I J}-\frac{1}{2} N^{m n}\left(\nabla_{\alpha} \tilde{F}_{m n}\right)^{I J}\right),
\end{gathered}
$$

where $\left(\nabla_{\alpha} s\right)^{I J}=D_{\alpha} s^{I J}+g\left(s^{I K} \tilde{A}_{\alpha}^{K J}-s^{J K} \tilde{A}_{\alpha}^{K I}\right)$ and the equations of motion $\dot{\theta}^{\alpha}=$ $-\frac{i g}{2} \eta_{I} \eta_{J} \tilde{W}^{\alpha I J}$ and $P^{m}=\Pi^{m}$ have been used. So using the definitions of (4.5),

$$
\frac{\partial}{\partial \tau}\left(\lambda^{\alpha} d_{\alpha}\right)=-\frac{i g}{4} \eta_{I} \eta_{J} \lambda^{\alpha} N^{m n}\left(\nabla_{\alpha} \tilde{F}_{m n}\right)^{I J}
$$

But $F_{m n}^{I J}$ satisfies $\left(\nabla_{\alpha} \tilde{F}_{m n}\right)^{I J}=\frac{i}{8} \gamma_{\alpha \beta[m}\left(\nabla_{n]} W^{\beta}\right)^{I J}$ where $\left(\nabla_{n} s\right)^{I J}=D_{n} s^{I J}+g\left(s^{I K} \tilde{B}_{n}^{K J}-\right.$ $\left.s^{J K} \tilde{B}_{n}^{K I}\right)$. Also, one can check that $N^{m n}=\frac{1}{2} \lambda \gamma^{m n} w$ where $w_{\alpha}$ is an anti-Weyl spinor with $S U(5)$ components

$$
w_{+}=\beta, \quad w^{a b}=v^{a b}, \quad w_{a}=0 .
$$

So

$$
\lambda^{\alpha} N^{m n}\left(\nabla_{\alpha} \tilde{F}_{m n}\right)^{I J}=\frac{i}{16} \lambda^{\alpha}\left(\lambda \gamma^{m n} w\right) \gamma_{\alpha \beta[m}\left(\nabla_{n]} \tilde{W}^{\beta}\right)^{I J}=-\frac{i}{8}\left(\lambda^{\delta} w_{\delta}\right) \lambda^{\alpha} \gamma_{\alpha \beta}^{n}\left(\nabla_{n} \tilde{W}^{\beta}\right)^{I J}
$$


using (5.2) and the gamma-matrix identity that $\eta_{m n} \gamma_{(\alpha \beta}^{m} \gamma_{\delta) \kappa}^{n}=0$. But one now can use that $\gamma_{\alpha \beta}^{n}\left(\nabla_{n} \tilde{W}^{\beta}\right)^{I J}=0$ to imply that (5.20) vanishes, and therefore $\lambda^{\alpha} d_{\alpha}$ is conserved.

Finally, it will be shown that $Q\left(\eta_{I} \eta_{J} \Psi^{I J}\right)=0$ gives the correct equation of motion in the presence of the background gauge field $\tilde{A}_{\alpha}^{I J}$. Since the canonical momentum for $\theta^{\alpha}$ and $x^{m}$ are

$$
\begin{gathered}
\frac{\partial L}{\partial \dot{\theta}^{\alpha}}=p_{\alpha}-\frac{i g}{2} \eta_{I} \eta_{J}\left(\tilde{A}_{\alpha}^{I J}-\frac{i}{2} \theta^{\beta} \gamma_{\alpha \beta}^{m} \tilde{B}_{m}^{I J}\right), \\
\frac{\partial L}{\partial \dot{x}^{m}}=P_{m}-\frac{i g}{2} \eta_{I} \eta_{J} \tilde{B}_{m}^{I J}
\end{gathered}
$$

one finds that

$$
\begin{gathered}
Q=\lambda^{\alpha} d_{\alpha}=\lambda^{\alpha}\left(p_{\alpha}+\frac{i}{2} P_{m} \gamma_{\alpha \beta}^{m} \theta^{\beta}\right) \\
=\lambda^{\alpha}\left(\frac{\partial L}{\partial \dot{\theta}^{\alpha}}+\frac{i}{2} \frac{\partial L}{\partial \dot{x}^{m}} \gamma_{\alpha \beta}^{m} \theta^{\beta}+\frac{i g}{2} \eta_{I} \eta_{J} \tilde{A}_{\alpha}^{I J}\right) .
\end{gathered}
$$

So

$Q\left(\eta_{I} \eta_{J} \Psi^{I J}\right)=-i \eta_{I} \eta_{J}\left(\lambda^{\alpha}\left(\nabla_{\alpha} C\right)^{I J}+\lambda^{\alpha} \lambda^{\beta}\left(\nabla_{\alpha} A_{\beta}\right)^{I J}+\lambda^{\alpha}\left(\lambda \gamma^{m n p q r} \lambda\right)\left(\nabla_{\alpha} A_{m n p q r}^{*}\right)^{I J}+\ldots\right)$

where $\left(\nabla_{\alpha} s\right)^{I J}=D_{\alpha} s^{I J}+g\left(s^{I K} \tilde{A}_{\alpha}^{K J}-s^{J K} \tilde{A}_{\alpha}^{K I}\right)$, which correctly covariantizes the supersymmetric derivatives with respect to the background gauge field.

\section{Appendix: Superparticle Cohomology at Zero Momentum}

In this appendix, the zero momentum cohomology of $Q=\lambda^{\alpha} d_{\alpha}$ will be computed for arbitrary ghost number and shown to correspond to the ghost, gluon, gluino, antigluino, antigluon, and antighost of super-Yang-Mills. Since $Q=\lambda^{\alpha} p_{\alpha}$ when $P_{m}=0$, the only reason one has non-trivial cohomology is because $\lambda^{\alpha}$ is constrained by (5.2). It will now be proven that the cohomology of $Q=\lambda^{\alpha} p_{\alpha}$ with constrained $\lambda^{\alpha}$ is equivalent to the "linear" cohomology of $\widehat{Q}$ with unconstrained $\lambda^{\alpha}$ where

$$
\begin{gathered}
\widehat{Q}=\lambda^{\alpha} p_{\alpha}+\left(\lambda \gamma^{m} \lambda\right) b_{m}+c^{m}\left(\lambda \gamma_{m} f\right)+\left(\lambda \gamma_{m} \lambda\right)\left(j \gamma^{m} g\right)-2\left(j_{\alpha} \lambda^{\alpha}\right)\left(g_{\beta} \lambda^{\beta}\right) \\
+\left(k \gamma_{m} \lambda\right) r^{m}+\left(\lambda \gamma^{m} \lambda\right) s_{m} t
\end{gathered}
$$

and "linear" cohomology signifies elements in the cohomology of $\widehat{Q}$ which are at most linearly dependent on the new variables $\left(c^{m}, g_{\alpha}, k^{\alpha}, s_{m}, u\right)$. Note that $\left(c^{m}, b_{m}\right),\left(g_{\alpha}, f^{\alpha}\right)$, $\left(k^{\alpha}, j_{\alpha}\right),\left(s_{m}, r^{m}\right)$, and $(u, t)$ are pairs of new variables and their conjugate momentum which have been added to the Hilbert space. The pairs $\left(c^{m}, b_{m}\right),\left(k^{\alpha}, j_{\alpha}\right)$ and $(u, t)$ are fermions of ghost-number $(1,-1),(2,-2)$ and $(3,-3)$ respectively, and the pairs $\left(g_{\alpha}, f^{\alpha}\right)$ and $\left(s_{m}, r^{m}\right)$ are bosons of ghost-number $(1,-1)$ and $(2,-2)$ respectively. 


\subsection{Equivalence of $Q$ and $\widehat{Q}$ cohomologies}

To relate the cohomologies of $Q$ and $\widehat{Q}$, consider a state $F(\lambda, \theta)$ in the cohomology of $Q$ with constrained $\lambda^{\alpha}$. Then $Q F=\left(\lambda \gamma^{m} \lambda\right) \tau_{m}$ for some $\tau_{m}$. So $\widehat{Q}\left(F-c^{m} \tau_{m}\right)=c^{m} Q \tau_{m}$. But $Q^{2} F=0$ implies that $\left(\lambda \gamma^{m} \lambda\right) Q \tau_{m}=0$, which implies that $Q \tau_{m}=\lambda \gamma_{m} \psi$ for some $\psi^{\alpha}$. So $\widehat{Q}\left(F-c^{m} \tau_{m}-g_{\alpha} \psi^{\alpha}\right)=-g_{\alpha} Q \psi^{\alpha}$. But $Q^{2} \tau_{m}=0$ implies that $\lambda \gamma_{m} Q \psi=0$, which implies that $Q \psi^{\alpha}=\left(\lambda \gamma^{n} \lambda\right)\left(\gamma_{n} \rho\right)^{\alpha}-2 \lambda^{\alpha}\left(\lambda^{\beta} \rho_{\beta}\right)$ for some $\rho_{\beta}$. This line of argument continues until one has $\widehat{Q}\left(F-c^{m} \tau_{m}-g_{\alpha} \psi^{\alpha}+k^{\alpha} \rho_{\alpha}+s_{m} \sigma^{m}+u \kappa\right)=u Q \kappa$. Finally, $Q^{2} \sigma^{m}=0$ implies that $\left(\lambda \gamma^{m} \lambda\right) Q \kappa=0$, which implies that $Q \kappa=0$. So for any state $F(\lambda, \theta)$ in the cohomology of $Q$ with constrained $\lambda^{\alpha}$, one can construct a state $\widehat{F}$ annihilated by $\widehat{Q}$ which is at most linear in $\left(c^{m}, g_{\alpha}, k^{\alpha}, s_{m}, u\right)$.

To show that $\widehat{F}$ is in the cohomology of $\widehat{Q}$, suppose that $\widehat{F}=\widehat{Q} \widehat{\Omega}$ for some $\widehat{\Omega}=$ $\Omega(\lambda, \theta)+c^{m} \xi_{m}(\lambda, \theta)+\ldots$ Then since $F$ is the term in $\widehat{F}$ which is independent of the new variables, $F=Q \Omega+\left(\lambda \gamma^{m} \lambda\right) \xi_{m}$. But this is not possible if $F$ is in the cohomology of $Q$ with constrained $\lambda^{\alpha}$, so $\widehat{F} \neq \widehat{Q} \widehat{\Omega}$ for any $\widehat{\Omega}$.

Now suppose that one starts with a state $\widehat{F}$ in the cohomology of $\widehat{Q}$ which is at most linear in $\left(c^{m}, g_{\alpha}, k^{\alpha}, s_{m}, u\right)$, i.e.

$$
\widehat{F}=F(\lambda, \theta)+c^{m} \tau_{m}(\lambda, \theta)+g_{\alpha} \psi^{\alpha}(\lambda, \theta)+k^{\alpha} \rho_{\alpha}(\lambda, \theta)+s_{m} \sigma^{m}(\lambda, \theta)+u \kappa(\lambda, \theta) .
$$

Then $\widehat{Q} \widehat{F}=0$ implies that $Q F=-\left(\lambda \gamma^{m} \lambda\right) \tau_{m}$, so $F$ is annihilated by $Q$ with constrained $\lambda^{\alpha}$.

To show that $F$ is in the cohomology of $Q$, suppose that $F=Q \Omega+\left(\lambda \gamma^{m} \lambda\right) \xi_{m}$ for some $\Omega(\lambda, \theta)$ and $\xi_{m}(\lambda, \theta)$. Then $\widehat{F}=\widehat{Q}\left(\Omega+c^{m} \xi_{m}\right)+c^{m}\left(\tau_{m}+Q \xi_{m}\right)+g_{\alpha} \psi^{\alpha}+\ldots$ So $\widehat{Q} \widehat{F}=0$ implies that $\left(\lambda \gamma^{m} \lambda\right)\left(\tau_{m}+Q \xi_{m}\right)=0$, which implies that $\tau_{m}+Q \xi_{m}=\lambda \gamma^{m} \chi$ for some $\chi^{\alpha}$. So $\widehat{F}=\widehat{Q}\left(\Omega+c^{m} \xi_{m}+g_{\alpha} \chi^{\alpha}\right)+g_{\alpha}\left(\psi^{\alpha}-Q \chi^{\alpha}\right)+k^{\alpha} \rho_{\alpha}+\ldots$. This argument continues until one finds that $\widehat{F}=\widehat{Q}\left(\Omega+c^{m} \xi_{m}+g_{\alpha} \chi^{\alpha}+\ldots+u \varepsilon\right)$, which is not possible if $\widehat{F}$ is in the cohomology of $\widehat{Q}$. So it has been proven that the cohomology of $Q$ with constrained $\lambda^{\alpha}$ is equivalent to the "linear" cohomology of $\widehat{Q}$ with unconstrained $\lambda^{\alpha}$.

\subsection{Evaluation of $\widehat{Q}$ cohomology}

Since $\widehat{Q}=\lambda^{\alpha} \widehat{p}_{\alpha}$ where $\lambda^{\alpha}$ is unconstrained and

$$
\widehat{p}_{\alpha}=p_{\alpha}+\left(\gamma^{m} \lambda\right)_{\alpha} b_{m}+c^{m}\left(\gamma_{m} f\right)_{\alpha}+\left(\gamma_{m} \lambda\right)_{\alpha}\left(j \gamma^{m} g\right)-2 j_{\alpha}\left(g_{\beta} \lambda^{\beta}\right)+\left(\gamma_{m} k\right)_{\alpha} r^{m}+\left(\gamma^{m} \lambda\right)_{\alpha} s_{m} t
$$


it is easy to evaluate the cohomology of $\widehat{Q}$. Using the quartet mechanism, one can choose a gauge such that states in the cohomology are independent of $\lambda^{\alpha}$ and $\theta^{\alpha}$. So states in the "linear" cohomology are represented by the elements $\left(1, c^{m}, g_{\alpha}, k^{\alpha}, s_{m}, u\right)$, which have ghost-number $(0,1,1,2,2,3)$ respectively.

To relate these elements to states in the cohomology of $Q$, one needs to find gaugeinvariant version of these elements which commute with $\widehat{Q}$. For example, $c^{m}-i \lambda \gamma^{m} \theta$ and $g_{\alpha}-i\left(\theta \gamma_{m}\right)_{\alpha} c^{m}+\frac{2}{3}\left(\lambda \gamma_{m} \theta\right)\left(\gamma^{m} \theta\right)_{\alpha}$ commute with $\widehat{Q}$, so $\lambda \gamma^{m} \theta$ and $\left(\lambda \gamma_{m} \theta\right)\left(\gamma^{m} \theta\right)_{\alpha}$ are the states in the cohomology of $Q$ which are associated with $c^{m}$ and $g_{\alpha}$. Similarly, one can show that $\left(\lambda \gamma^{m} \theta\right)\left(\lambda \gamma^{n} \theta\right)\left(\theta \gamma_{m n}\right)^{\alpha}$ is the state associated with $k^{\alpha},\left(\lambda \gamma^{m} \theta\right)\left(\lambda \gamma^{n} \theta\right)\left(\theta \gamma_{m n p} \theta\right)$ is the state associated with $s_{p}$, and $\left(\lambda \gamma^{m} \theta\right)\left(\lambda \gamma^{n} \theta\right)\left(\lambda \gamma^{p} \theta\right)\left(\theta \gamma_{m n p} \theta\right)$ is the state associated with $u$.

Comparing these states with the superfields in (5.8), one finds that the zero momentum states in the cohomology of $Q$ correspond to the ghost, gluon, gluino, antigluino, antigluon, and antighost of super-Yang-Mills.

Acknowledgements: I would like to thank Warren Siegel for useful discussions, João Barcelos-Neto for suggesting that these notes be written up, and the Clay Mathematics Institute, CNPq grant 300256/94-9, Pronex 66.2002/1998-9, and FAPESP grant 99/127630 for partial financial support. This research was partially conducted during the period the author was employed by the Clay Mathematics Institute as a CMI Prize Fellow. 


\section{References}

[1] N. Berkovits, JHEP 04 (2000) 018, hep-th/0001035;

N. Berkovits, hep-th/0008145.

[2] E. Witten, hep-th/9207094.

[3] L. Brink and J.H. Schwarz, Phys. Lett. 100B (1981) 310.

[4] W. Siegel, Phys. Lett. 128B (1983) 397.

[5] M.B. Green and J.H. Schwarz, Nucl. Phys. B181 (1981) 502.

[6] I.A. Batalin and G.A. Vilkovisky, Phys. Rev. D28 (1983) 2567.

[7] D. Friedan and P. Windey, Nucl. Phys. B235 (1984) 395.

[8] W. Siegel, Phys. Lett. 80B (1979) 220.

[9] P. Howe, Phys. Lett. B258 (1991) 141.

[10] N. Berkovits, JHEP 09 (2000) 046, hep-th/0006003. 